\title{
El registro de mayor altitud del Hurón Mayor Galictis vittata, con notas sobre su presencia y conservación dentro del departamento de Caldas, en la región andina de Colombia
}

\author{
Sergio Escobar-Lasso ${ }^{1,2^{*}}$ y Christian F. Guzmán-Hernández ${ }^{3,4}$
}

Introduction: The Greater Grison Galictis vittata is distributed in the northern part of Latin America, from southern Mexico through Central America to reach northern Bolivia and Brazil, from sea level to 1.500 $\mathrm{m}$, being more common below $500 \mathrm{~m}$. In the department of Caldas, located in the Central Cordillera of Colombia, there is no literature to collect information of G. vittata. Therefore, the aim of this paper is to present the record of greater height of the species, conduct a review of the distribution of $G$. vittata within the Caldas department, and investigate about threats to their populations in that region.

Materials and methods: To carry out the review of the distribution and conservation of G. vittata within the Caldas department, were taken into account: A) Specimens, or part of these, deposited within biological collections. B) Records in scientific literature. C) Photographic records. D) Records of live individuals coming from the Caldas department who entered to the Center of Care, Assessment and Rehabilitation of Wildlife of the Regional Autonomous Corporation of Caldas (Corpocaldas).

Results and discussion: To date, there are records in the municipalities of Manizales, Marquetalia, and Villamaría, between 900 and 2,200 m. In this work, we determined that this was the highest elevation that has been reported to date for this species not only in Colombia but throughout all its range of distribution. Its main threats within the Caldas department are illegal hunting and habitat loss. In the department of Caldas, the majority of the records are concentrated on the banks of the Guarino River, in the municipality of Marquetalia. We proposed that the area of the Guarino River, could be protected to prevent the local extinction of G. vittata in that department.

Key words: Andean forest, habitat loss, illegal hunting, local distribution.

\section{Resumen}

Realizamos una revisión de la distribución y amenazas del Hurón Mayor Galictis vittata dentro del departamento de Caldas, en la región andina de Colombia. Hasta la fecha, hay registros en los municipios de Manizales, Marquetalia, y Villamaría, entre los 900 y los 2,200 m. Encontramos que esta última altura corresponde a la mayor altitud de esta especie tanto en Colombia como a través de su intervalo de distribución. Su principal amenaza dentro del departamento es la cacería ilegal y la pérdida de hábitat.

\footnotetext{
${ }^{1}$ Fundación R.A.N.A (Restauración de Ambientes Neotropicales Alterados). Calle 6623 b 56, Manizales. Caldas, Colombia 17001000. E-mail: biosergiobike@gmail.com (SE-L)

2Programa de Posgrado en Conservación y Manejo de Vida Silvestre, Instituto Internacional de Conservación y Manejo de Vida Silvestre. ICOMVIS. Heredia, Costa Rica.

${ }^{3}$ Semillero de Investigación en Mastozoología (SIMas), Programa de Biología, Universidad de Caldas. Manizales, Colombia. E-mail: christian.1710514860@ucaldas.edu.co (CFGG-H)

${ }^{4}$ Grupo de Genética, Biodiversidad y manejo de Ecosistemas (GEBIOME), Universidad de Caldas. Manizales, Colombia. ${ }^{*}$ Corresponding autor
} 
Palabras clave: bosques andinos, cacería ilegal, distribución local, pérdida de hábitat.

Introducción

Como lo corroboran recientes estudios morfológicos, biogeográficos y moleculares para el género Galictis se reconocen dos especies: G. cuja (Hurón Menor) y G. vittata (Hurón mayor; Bornholdt et al. 2013). Galictis cuja se distribuye en el sur de América Latina, desde el extremo sur de Perú, el sur de Bolivia y el noreste de Brasil hasta el sur de Chile y Argentina; en contraste G. vittata se distribuye en la parte norte, desde el sur de México atraviesa todo Centro América hasta llegar al norte de Bolivia y Brasil (Yensen y Tarifa 2003; Cuarón et al. 2008; Bornholdt et al. 2013). Esta última especie es considerada una rareza demográfica, ya que tiene una densidad baja a lo largo de su intervalo de distribución (Yensen y Tarifa 2003; Cuarón et al. 2008).

El Hurón Mayor G. vittata tiene un cuerpo alargado y delgado, sus patas son cortas con garras afiladas y levemente palmeadas, tiene una cola pequeña y peluda (Yensen y Tarifa 2003). Su cuello es alargado y su cabeza es pequeña y aplanada, con orejas pequeñas, anchas y redondeadas; los ojos tienen un iris de color café o negro (Yensen y Tarifa 2003), con una reflexión azul brillante en la noche (Kaufmann y Kaufmann 1965).

El dorso de la cabeza, la espalda, los costados y la cola son de apariencia canosa "color gris" debido a que los pelos son negros con la punta blanca; el rostro, la garganta, el vientre y las extremidades suelen ser de color negro sólido; una franja de color blanco recorre la frente hasta llegar a los hombros, la cual separa el gris dorsal del negro ventral (Yensen y Tarifa 2003). G. vittata tienen una actividad principalmente diurna (Kaufmann y Kaufmann 1965), pero también es activo en la noche; son solitarios, formando en ocasiones grupos familiares discretos (Yensen y Tarifa 2003). Generalmente se refugia en oquedades de árboles y en cuevas abandonadas por armadillos, habita en las cercanías de ríos, quebradas y riachuelos, ya que es una especie de hábitos asociados al agua (Linares 1998). Es considerada una especie omnívora y en ocasiones ataca aves de corral en áreas rurales (Nowak 2005; Cuarón et al. 2008).

Altitudinalmente G. vittata ocurre desde el nivel del mar hasta los 1,500 m, siendo más común por debajo de los 500 m (Timm et al. 1989; Yensen y Tarifa 2003; Cuarón et al. 2008; Monjeau et al. 2009; Bornholdt et al. 2013). En Colombia su distribución es poco conocida; de acuerdo con la lista de mamíferos publicada por Alberico et al. (2000) esta especie ocurre en los departamentos de Antioquia, Caldas, Casanare, Cauca, Cundinamarca, Magdalena, Meta, Nariño, Sucre y Vichada, desde el nivel del mar hasta los 1,200 m, registros igualmente soportados a posteriori por Cuartas-Calle y Muñoz-Arango (2003), Castaño (2012), Ramírez-Chaves y Noguera-Urbano (2010), Ramírez-Chaves y Pérez (2010), y Solari et al. (2013).

Específicamente para el departamento de Caldas los trabajos de Castaño (2012) y Escobar-Lasso et al. (2013), son los únicos que reportan avistamientos de G. vittata. Sin embargo, éstos no documentan registros de ejemplares depositados en colecciones científicas o fotografías publicadas que corroboren las observaciones. De esta manera, 
no se cuenta con publicaciones que recopilen mayor información sobre G. vittata en el departamento.

Considerando lo anterior, el objetivo de este trabajo es dar a conocer el registro de mayor altura para la especie, realizar una revisión de la distribución de G. vittata dentro del departamento de Caldas, e indagar sobre las amenazas de sus poblaciones en esta región.

El Departamento de Caldas está ubicado en la parte centro occidental de la región andina de Colombia (Fig. 1). Sus límites se extienden desde la cuenca del río Magdalena hasta la cuenca del río Cauca, incluyendo ambas vertientes de la cordillera Central y la vertiente oriental de la cordillera Occidental (Instituto Geográfico Agustín Codazzi 1990; Castaño et al. 2003). El departamento de Caldas está compuesto por 12 cuencas hidrográficas y 27 municipios (Fig. 1), tiene una extensión de 7,457 km² que representan el $0.7 \%$ del total del país y presenta un intervalo altitudinal entre los 200 y los 5,423 m (Instituto Geográfico Agustín Codazzi 1990; Castaño 2012).

Figura 1. Registros del Hurón Mayor Galictis vittata en el departamento de Caldas, Cordillera Central de Colombia. Sobre el mapa se proyectan las sinco localidades donde se tienen registros confirmados. Note como la mayoría de los registros se concentran a las orillas del Río Guarinó, en el municipio de Marquetalia. Detalles de las localidades en Tabla 1.

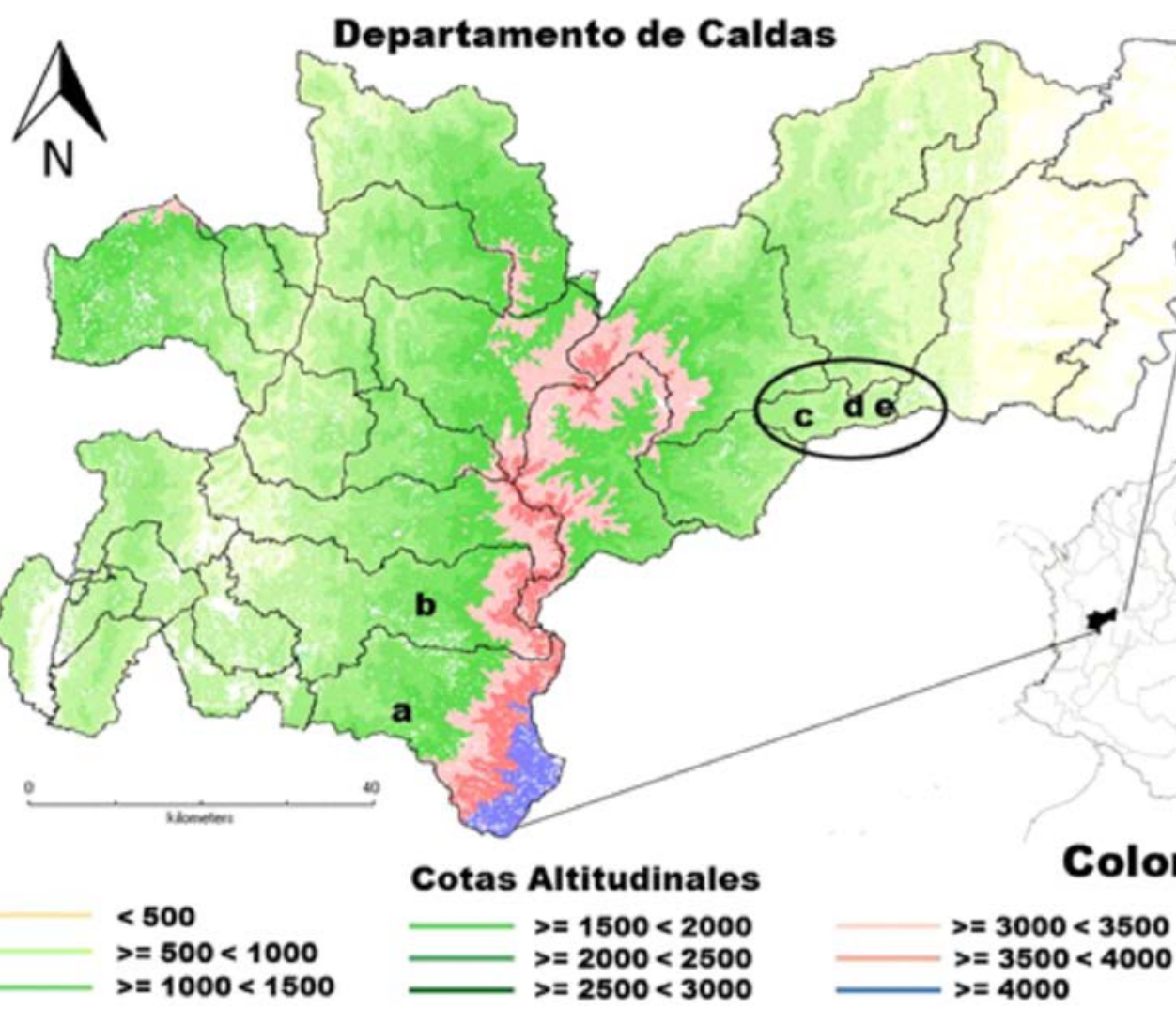

De las seis especies de mustélidos registradas para Colombia (Alberico et al. 2000; Ramírez-Chaves y Mantilla-Meluk 2009), en el departamento de Caldas se encuentran cuatro (66.6\%): Eira barbara (Tayra), Lontra longicaudis (Nutria), Mustela frenata (Comadreja) y Galictis vittata (Hurón; Castaño et al. 2003; Castaño 2012; Castaño y Corrales 2010; Escobar-Lasso et al. 2013), de éstas sólo se evalúo la distribución de G. vittata, en dicho departamento, a partir de especímenes depositados en la colección mastozoológica del Museo de Historia Natural de la Universidad de Caldas, Manizales- 
Colombia (MHN-UC), y registros fotográficos realizados in situ de individuos que ingresaron al Centro de Atención, Valoración y Rehabilitación de Fauna Silvestre (CAV) de Caldas; también se tuvo en cuenta los registros encontrados por Castaño (2012) y Escobar-Lasso et al. (2013). Se realizarón los mapas de las localidades con resultados de Hurón en el departamento (Fig. 1), para identificar posibles áreas con alta concentración de registros.

Para el departamento de Caldas se tienen registros de cinco individuos de G. Vittata (Tabla 1), pertenecientes a los municipios de Manizales, Marquetalia, y Villamaría, entre los 900 y los 2,200 m (Fig. 1 y 2), que al ser revisados en este trabajo se precisa que el individuo registrado en el municipio de Manizales a los 2,200 m (Fig. 2A), es el reporte de mayor altura de G. vittata para Colombia. Por lo tanto, el registro en la cordillera Central de Colombia y el reportado por Rumiz et al. (1998) en la vertiente oriental de la cordillera de los Andes en Bolivia a los 2,000 m. Se constituyen como los registros de mayor altura para la especie.

\begin{tabular}{|c|c|c|}
\hline Código Mapa & Localidad & Coordenadas \\
\hline a & $\begin{array}{l}\text { Individuo avistado a las cercanías de Río Claro, } \\
\text { Municipio de Villamaría (Escobar-Lasso et al. 2013). }\end{array}$ & $4^{\circ} 59^{\prime} 07.84^{\prime \prime} \mathrm{N},-75^{\circ} 33^{\prime} 1.7^{\prime \prime} \mathrm{W} ; 1,624 \mathrm{~m}$ \\
\hline b & $\begin{array}{l}\text { Individuo avistado en el } 2009 \text { en el Recinto del } \\
\text { Pensamiento, Municipio de Manizales (Fig. 2a). }\end{array}$ & $5^{\circ} 2^{\prime} 28.91^{\prime \prime} \mathrm{N},-75^{\circ} 26^{\prime} 48.17^{\prime \prime} \mathrm{W} ; 2,200 \mathrm{~m}$ \\
\hline c & $\begin{array}{l}\text { Individuo ingresado en el } 2009 \text { al Centro de Atención, } \\
\text { Valoración y Rehabilitación de Fauna Silvestre } \\
\text { (CAV) de Caldas, proveniente de la zona urbana del } \\
\text { municipio de Marquetalia, sector barrio La Castilla } \\
\text { (ver Fig. } 2 \text { b). }\end{array}$ & $5^{\circ} 17^{\prime} 46.88^{\prime \prime} \mathrm{N},-75^{\circ} 03^{\prime} 20.63^{\prime \prime} \mathrm{W} ; 1,600 \mathrm{~m}$ \\
\hline$d$ & $\begin{array}{l}\text { Individuo registrados en el } 2009 \text { a las orillas del río } \\
\text { Guarinó, en el municipio de Marquetalia, límites con } \\
\text { el municipio de Victoria (MHN-UC 1156). }\end{array}$ & $5^{\circ} 17^{\prime} 26.06^{\prime \prime} \mathrm{N},-75^{\circ} 00^{\prime} 27.57^{\prime \prime} \mathrm{W} ; 900 \mathrm{~m}$ \\
\hline e & $\begin{array}{l}\text { Individuo ingresado en el } 2009 \text { al Centro de Atención, } \\
\text { Valoración y Rehabilitación de Fauna Silvestre (CAV) } \\
\text { de Caldas, proveniente del río Guarinó, Municipio de } \\
\text { Marquetalia (Fig. 2c). }\end{array}$ & $5^{\circ} 17^{\prime} 26.06^{\prime \prime} \mathrm{N},-75^{\circ} 00^{\prime} 27.57^{\prime \prime} \mathrm{W} ; 900 \mathrm{~m}$ \\
\hline
\end{tabular}

De los cinco individuos registrados (Fig. 1) tres provienen de decomisos a cazadores (Fig. 2B, C y MHN-UC 1156), problemática que ya ha sido documentada para la región debido a la fuerte presión antrópica por causa de la cacería ilegal y uso como mascotas, poniendo en riesgo la supervivencia de la especie en diferentes lugares donde se encuentra (Yensen y Tarifa 2003; Cuarón et al. 2008).

Al mapear las localidades donde se tienen registros confirmados de G. vittata en el departamento de Caldas, se puede ver que hay registros en ambos flancos (occidental y oriental) de la Cordillera Central (Fig. 1). Sin embargo, la mayoría de los registros se concentran a las orillas del Río Guarinó, en el municipio de Marquetalia. Considerando lo anterior se propone que el área del río Guarinó sea protegida para evitar la extinción local de G. vittata en dicho departamento, aspecto que debería ser considerado como componente importante a la hora de generar planes de conservación del Hurón en el departamento de Caldas y otras áreas de Colombia. 
Figura $2 . \quad$ Registros fotográficos del Hurón Mayor Galictis vittata en el departamento de Caldas-Colombia.

(a), Individuo avistado en el 2009 en el Recinto del Pensamiento, Municipio de Manizales. (b) Individuo ingresado al Centro de Atención, Valoración y Rehabilitación de Fauna Silvestre (CAV) en el 2009, proveniente de la zona urbana del municipio de Marquetalia. (c) Individuo ingresado al Centro de Atención, Valoración y Rehabilitación de Fauna Silvestre (CAV) en el 2009, proveniente del Río Guarinó, municipio Marquetalia. Fotos: Oscar Ospina-Herrera.

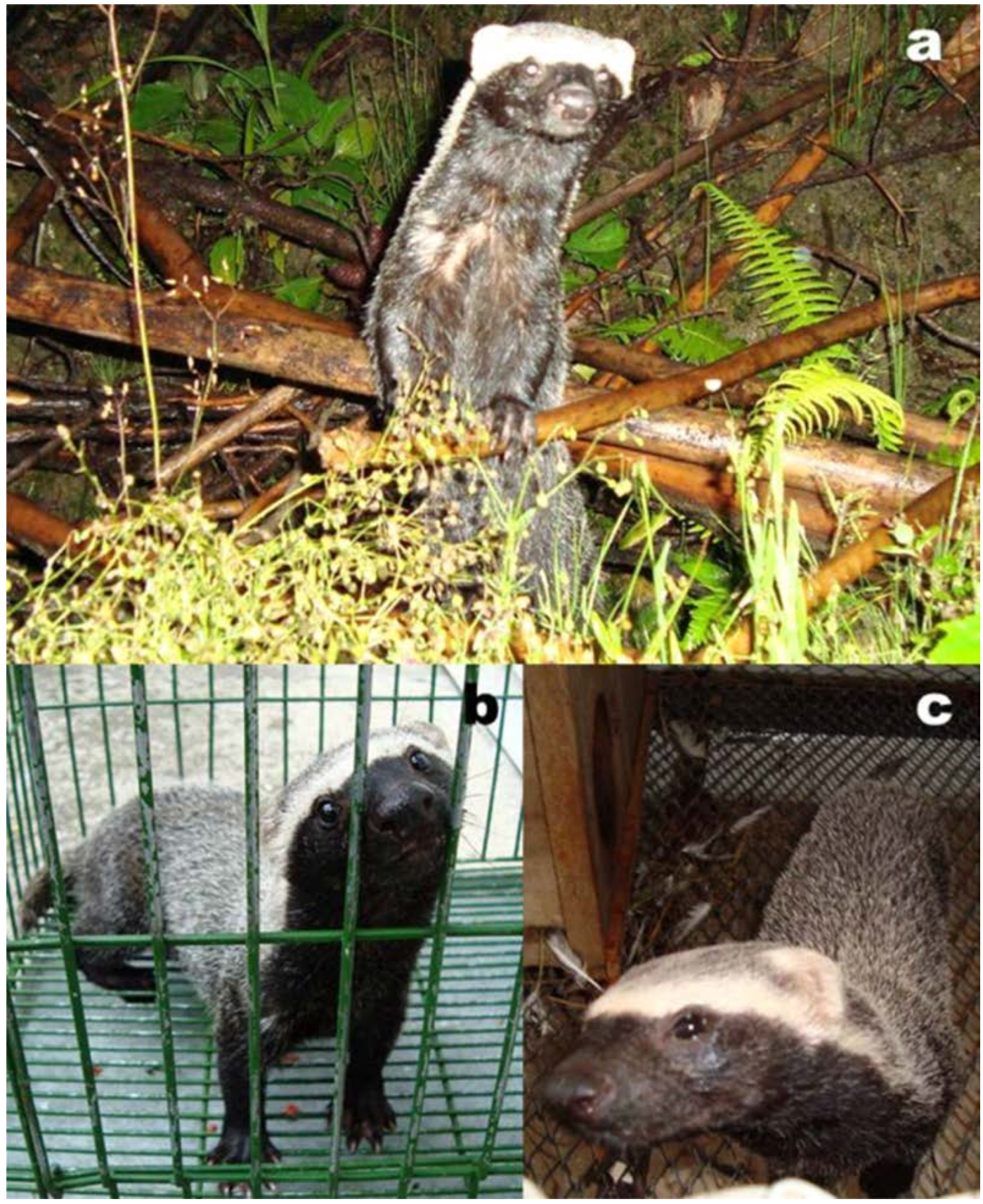

Deseamos expresar nuestros más sinceros agradecimientos a J. Salazar-Escobar curador del Museo de Historia Natural de la Universidad de Caldas (Colombia) por permitirnos el acceso a la colección de mamíferos bajo su cuidado. A O. Ospina-Herrera coordinador de fauna silvestre de la Corporación Autónoma Regional de Caldas (Corpocaldas) por compartir sus fotografías y registros con nosotros. A T. Lasso-Amézquita por facilitar las instalaciones y recursos del Observatorio de Conflictos Ambientales (OCA) de la Universidad de Caldas, para la preparación del presente manuscrito. 
Alberico, M., A. Cadena, J. Hernández-Camacho, y Y. Muñoz-Saba. 2000. Mamíferos (Synapsida: Theria) de Colombia. Biota Colombiana 1:43-75.

Bornholdt, R., K. Helgen, K. Klaus-Peter, L. Oliveira, M. Lucherini, y E. Eizirik. 2013. Taxonomic revision of the genus Galictis (Carnivora: Mustelidae): species delimitation, morphological diagnosis, and refined mapping of geographical distribution. Zoological Journal of the Linnean Society 167:449-472.

Cabrera, A. 1958. Catálogo de los Mamíferos de América del Sur. Revista del Museo Argentino de Ciencias Naturales "Bernardino Rivadavia" e Instituto Nacional de Investigación de las Ciencias Naturales, Zoología 4:1-305.

Castaño, J. H. 2012. Mamíferos de Caldas: un análisis de vacíos de información. Boletín Científico Museo de Historia Natural Universidad de Caldas 16:101-119.

Castaño, J. H., y J. D. Corrales. 2010. Mamíferos de la cuenca del río La Miel (Caldas): diversidad y uso cultural. Boletín Científico Museo de Historia Natural Universidad de Caldas 14:56-75.

Castaño, J. H., Y. Muñoz-Saba, J. H. Botero, y J. H. Vélez. 2003. Mamíferos del departamento de Caldas-Colombia. Biota Colombiana 4:247-259.

Cuarón, A. D., F. Reid, y K. Helgen. 2008. Galictis vittata. In IUCN 2013. IUCN Red List of Threatened Species. Version 2013.1.

Cuartas-Calle, C. A., y J. Muñoz-Arango. 2003. Listado de mamíferos (Mammalia: Theria) del departamento de Antioquia, Colombia. Biota Colombiana 4:65-78.

Escobar-Lasso, S., J. Cerón-Cardona, y J. H. Castaño. 2013. Los mamíferos de la cuenca del río Chinchiná, en la región andina de Colombia. Therya 4:139-155.

KaufmanN, J. H., y A. Kaufmann. 1965. Observations of the behavior of tayras and grisons. Zeitschrift fur Saugetierkunde 30:146-155.

Lasso-Amézquita, T., F. Sánchez-Zapata, y J. G. Valencia-Hernández. 2008. El derecho de una región al agua. Transvase del río Guarinó al río la Miel. Editorial Universidad de Caldas. Manizales, Colombia.

Linares, O. J. 1998. Mamíferos de Venezuela. Sociedad Conservacionista Audubon de Venezuela. Caracas, Venezuela.

Monjeau, J. A., J. A. Tort, J. Márquez, P. Jayat, B. N. Palmer-Fry, S. D. Nazar-Anchorena, A. D. Vincenzo, And F. Polop. 2009. Latitudinal patterns of species richness distribution in South American carnivores. Mastozoología Neotropical 16:1-14.

Nowak, R. M. 2005. Walker's Carnivores of the world. Johns Hopkins University Press. Baltimore, EE.UU.

Ramírez-Chaves, H. E., y H. Mantilla-Meluk. 2009. Nuevo registro de la Comadreja Colombiana Mustela felipei (Carnivora: Mustelidae), con notas sobre su distribución y conservación. Mastozoología Neotropical 16:379-388.

Ramírez-Chaves, H. E., y E. A. Noguera-Urbano. 2010. Lista preliminar de los mamíferos (Mammalia: Theria) del departamento de Nariño, Colombia. Biota Colombiana 11:117-140.

Ramírez-Chaves, H. E., y W. A. Pérez. 2010. Mamíferos (Mammalia: Theria) del departamento del Cauca, Colombia. Biota Colombiana 11:141-171. 
Rumiz, D. I., C. F. Eulert, y R. ArisPe. 1998. Evaluación de la diversidad de mamíferos medianos y grandes en el Parque Nacional Carrasco (Cochabamba, Bolivia). Revista Boliviana de Ecología 4:77-90.

Solari, S., Y. Muñoz-Saba, J. V. Rodríguez-Mahecha, T. R. Defler, H. E. Ramírez-Chaves y F. Trujılo. 2013. Riqueza, Endemismo y Conservación de los Mamíferos de Colombia. Mastozoología Neotropical 20:301-365.

Timm, R. M., D. E. Wilson, B. L. Clauson, R. K. Laval, and C. S. Vaughan. 1989. Mammals of the La Selva-Braulio Carrillo complex, Costa Rica. North American Fauna 75:1162.

Yensen, E., AND T. Tarifa. 2003. Galictis vittata. Mammalian Species 727:1-8.

Sometido: 17 de septiembre de 2013

Revisado: 23 de julio de 2014

Aceptado: 1 de agosto de 2014

Editor asociado: Jesús Maldonado

Diseño gráfico editorial: Gerardo Hernández 
